\title{
Pulmonary Tuberculosis Confirmed by Percutaneous Transthoracic Needle Biopsy: Analysis of CT Findings and Review of Correlations with Underlying Lung Disease
}

\author{
Ji Young Choo ${ }^{1}$, Ki Yeol Lee ${ }^{1}$, Mi-young Kim², Eun-young Kang ${ }^{1}$, Yu Whan Oh${ }^{1}$, Seung Hwa Lee ${ }^{1}$, \\ Bo Kyung Seo ${ }^{1}$, Bo Kyung Je ${ }^{1}$ \\ 'Department of Radiology, Korea University College of Medicine, Seoul, South Korea \\ ${ }^{2}$ Department of Radiology, Asan Medical Center, University of Ulsan College of Medicine, Seoul, South Korea
}

\begin{abstract}
Background: Pulmonary tuberculosis (TB) can produce unusual radiographic findings. Further, negative sputum and bronchoscopic results are common. Early diagnosis is equally as significant as treatment in the reduction of morbidity and mortality associated with pulmonary TB.

Aims: The aim of this study was to assess computed tomography (CT) findings of pulmonary TB, confirmed via percutaneous transthoracic needle biopsy (PTNB), and to correlate these findings with coexisting, underlying, lung diseases if present.

Study Design: Cross sectional study.

Methods: We selected eighty-four patients who were diagnosed with pulmonary TB by way of PTNB. Initially, acid-fast bacilli smear test results from these patients were negative. CT findings were reviewed to detect the presence of parenchymal abnormalities as follows: nodule(s) ( $<3 \mathrm{~cm}$ in diameter), mass (any masses $\geq 3$ $\mathrm{cm}$ ), daughter nodules, air-space consolidation, cavitation, calcification, lymphadenopathy, mediastinal lymphadenopathy, and associated lung parenchymal disease.
\end{abstract}

Results: The CT findings of pulmonary TB confirmed by PTNB included nodules in 44 of $84(52.4 \%)$ cases; 15 of these 44 cases (34.1\%) had daughter nodules. The second most common finding was masses in 24 cases $(28.6 \%)$, nine of which also had daughter nodules. 16 cases $(19.0 \%)$ displayed nonsegmental consolidation. Of these 16 cases, four had coexisting usual interstitial pneumonia; four others had emphysema. Two patients with a mass had underlying pneumoconiosis.

Conclusion: Nodules or a mass mimicking lung cancer were the most common findings on CT scans in patients with pulmonary $\mathrm{TB}$, confirmed via PTNB. The second most common finding was airspace consolidation. Therefore, PNTB is useful for the accurate diagnosis of pulmonary $\mathrm{TB}$ in the following cases: airspace consolidation or mass associated with underlying usual interstitial pneumonia, emphysema mimicking lung malignancy or cases of bacterial pneumonia. (Balkan Med J 2014;31:208-13).

Key Words: Percutaneous transthoracic needle biopsy, pulmonary, tuberculosis
Pulmonary tuberculosis (TB) is a common lung infection worldwide. Detection of acid-fast bacilli (AFB) in stained sputum typically allows for the diagnosis of pulmonary TB (1-3). However, AFB smear results may be negative, and early diagnosis is often difficult if the process is not discovered via radiological means or by a clinician. Early diagnosis is equally as significant as treatment in reducing the morbidity and mortality associated with pulmonary TB. Transthoracic biopsy and fine-needle aspiration have been demonstrated to be valuable for the diagnosis of tuberculosis $(3,4)$. Specifically, they are beneficial for patients who have unusual manifestations discovered via radiogra- phy. The incidence of pulmonary tuberculosis is higher in patients with chronic interstitial lung disease than in the general population. Furthermore, radiologic findings of TB are frequently atypical (5) and findings of patients with underlying emphysema have not been reported.

Our purpose in this study was therefore to assess computed tomography (CT) findings in patients with pulmonary TB, confirmed via percutaneous transthoracic needle biopsy (PTNB) with negative sputum, fiberoptic bronchoscopy and/ or bronchial lavage smear results. An additional aim was to determine the correlation between the CT findings of pulmonary $\mathrm{TB}$ and those of co-existing pulmonary diseases. 


\section{MATERIAL AND METHODS}

\section{Study subjects}

Approval was obtained from the institutional review board of our institution and full informed consent for the procedure was obtained from all patients. Eighty-four patients (average age, $59.1 \pm 9$ years; age range, 16 to 72 years) were enrolled in the study. These 84 patients consisted of 51 men and 33 women who were histologically diagnosed with pulmonary $\mathrm{TB}$, confirmed via positive polymerase chain reaction (PCR) results for PTNB samples. These patients underwent chest CT between January 2006 and December 2012. All patients underwent sputum examination and up to three consecutive specimens were obtained for acid fast staining in each case. We performed culture and PCR for M. tuberculosis using biopsy or aspiration specimens. We selected patients with histologically and PCR-confirmed TB (positive PCR for M. tuberculosis) to exclude nontuberculous mycobacterial infections.

\section{Imaging methods}

In 28 of the 84 patients, chest $\mathrm{CT}$ was performed using a 4-slice scanner (Somatom volume zoom; Siemens, Erlangen, Germany). In 56 patients, chest CT was performed using a 64-slice scanner (Brilliance 64; Philips, Cleveland, $\mathrm{OH}$, USA). For CT using the 4-slice scanner, scans were performed at $120 \mathrm{kVp}$ and $180 \mathrm{mAs}$, with $10-\mathrm{mm}$ collimation and $10-\mathrm{mm}$ reconstruction. For $\mathrm{CT}$ using the 64 -slice scanner, scans were performed at $120 \mathrm{kVp}$ and $250 \mathrm{mAs}$, with a pitch of 0.671 and collimation of 4-8 $\mathrm{mm}$ through the thorax at end-inspiration. The table feed was $7.5-12 \mathrm{~mm}$ per 0.75 -second rotation. Nonionic contrast material (Ultravist370; Schering, Berlin, Germany) was injected into the antebrachial vein $(130 \mathrm{~mL}$; flow rate, $4.0 \mathrm{~mL} / \mathrm{s}$ ) for the conventional chest CT scan in 69 patients.

\section{Analysis of chest CT scans}

Two chest radiologists (J.Y. C., K.Y. L.) with 5 and 13 years' experience, respectively, evaluated the chest CT scans. CT scans were viewed primarily in the transverse plane, but they were also viewed in the sagittal or coronal planes if available. Final decisions on the findings were reached by consensus. Patterns of parenchymal abnormalities on chest CT scans were as follows: nodule(s) $(<3 \mathrm{~cm}$ in diameter), mass (any masses ${ }^{3} 3 \mathrm{~cm}$ ), and air-space consolidation defined as a homogeneous increase in pulmonary parenchymal opacity that obscured the margins of vessels and airway walls. In addition, CT images were assessed for the following: diameter of mass or nodule; margin of mass or nodule (spiculated, lobulated, or smooth); presence of daughter nodule(s); presence of cavity; associated abnormalities such as mediastinal lymph node enlargement and pleural effusion; presence of calcifications in the lesion; location of the lesion, and coexisting pulmonary diseases such as emphysema, idiopathic interstitial pneumonia, pneumoconiosis, etc.

\section{PTNB method}

Percutaneous transthoracic needle biopsy was performed under CT $(\mathrm{n}=51)$, ultrasonography (US, $\mathrm{n}=9)$, or fluoroscopic guidance $(n=24)$ by chest radiologists (J.Y.C, Y.H.O., M.Y.K., K.Y.L). Each patient underwent PTNB at least once. Percutaneous transthoracic aspiration, in addition to PTNB, was performed in 24 patients. Aspiration was performed with a 22-gauge Chiba needle (M. I. Tech; Seoul, Korea), while a 19.5 gauge Autovac needle (BARD; Wachhausstrasse, Germany) was used for percutaneous cutting biopsies. In general, CT guidance was preferred, but fluoroscopic guidance was used in less cooperative patients or when CT was not available. US guidance was typically used in patients with a lesion attached to the pleura or chest wall. In all cases, lung aspirates and specimens from each biopsy sample were analysed in three different ways: histologic examination, PCR analysis, and/or culture.

Eight patients whose PTNB results were inadequate for diagnosis underwent repeat biopsy. Chest radiographs were obtained immediately after completion of PTNB and again 4 hours later to determine the presence of pneumothorax.

Histologic diagnosis and PCR for detecting M. tuberculosis Histopathological diagnosis of TB was based on chronic granulomatous inflammation, with or without caseous necrosis. We performed PCR on lung aspirates using an $M$. tuberculosis kit (Amplicor; Roche Diagnostic Systems, Somerville, NJ, USA) that targets a part of the 16S rRNA gene for amplification. The complete $M$. tuberculosis test was performed according to the manufacturer's instructions. Histopathological examination of tissue specimens and amplification of M. tuberculosis by PCR was performed for all 84 cases.

\section{RESULTS}

All 84 patients had positive TB PCR test results, as well as chronic granulomatous inflammation on histologic examination. Thirty-two patients had positive culture test results for M. tuberculosis.

The most common CT finding of pulmonary $\mathrm{TB}$, confirmed via PTNB, was a pulmonary nodule (44 cases, 52.4\%); daughter nodules were present in 15 cases $(34.0 \%)$. Nodules ranged in size from 1.2 to $2.8 \mathrm{~cm}$ in diameter. Margins of nodules were spiculated in 26 cases (59.1\%), lobulated in eight (18.2\%), and smooth in 10 cases $(22.7 \%)$. Additional daughter nodules in the same lobe were present in nine patients (20.4\%), while six patients $(13.6 \%)$ had nodules present in other lobes. 
TABLE 1. Tuberculosis in patients confirmed by PTNB: profiles of 84 patients

\begin{tabular}{|c|c|c|c|}
\hline & \multicolumn{3}{|c|}{ CT Findings, $\mathrm{n}(\%)$} \\
\hline & $\begin{array}{l}\begin{array}{l}\text { Nodule } \\
(\mathrm{n}=44, \\
52.4 \%)\end{array} \\
\end{array}$ & $\begin{array}{l}\text { Mass } \\
(\mathrm{n}=24, \\
28.6 \%)\end{array}$ & $\begin{array}{l}\text { Consolidation } \\
\qquad(\mathrm{n}=16, \\
19.0 \%)\end{array}$ \\
\hline Mean size $(\mathrm{cm})$ & $2.2(1.2-2.8)$ & $4.2(3.3-5.7)$ & \\
\hline \multicolumn{4}{|l|}{ Lesion margins } \\
\hline Spiculated & $26(59.1 \%)$ & $14(58.3 \%)$ & \\
\hline Lobulated & $8(18.2 \%)$ & $9(37.5 \%)$ & \\
\hline Smooth & $10(22.7 \%)$ & $1(4.2 \%)$ & \\
\hline \multicolumn{4}{|l|}{ Daughter nodules } \\
\hline Same lobe & $9(20.4 \%)$ & $3(12.5 \%)$ & $1(6.2 \%)$ \\
\hline Different lobe & $6(13.6 \%)$ & $6(25.0 \%)$ & $3(18.7 \%)$ \\
\hline Cavitation & $3(6.8 \%)$ & $8(33.3 \%)$ & $2(12.5 \%)$ \\
\hline $\begin{array}{l}\text { Mediastinal } \\
\text { lymphadenopathy }\end{array}$ & $1(2.3 \%)$ & $1(4.2 \%)$ & \\
\hline Calcification (eccentric) & $1(2.3 \%)$ & $1(4.2 \%)$ & \\
\hline \multicolumn{4}{|l|}{ Lesion location } \\
\hline Upper lobe & $24(54.5 \%)$ & $19(79.2 \%)$ & $9(56.2 \%)$ \\
\hline Lower lobe & $17(38.6 \%)$ & $4(16.7 \%)$ & $3(18.7 \%)$ \\
\hline Middle lobe & $3(6.8 \%)$ & $1(4.2 \%)$ & $3(18.7 \%)$ \\
\hline Right lung $^{\mathrm{a}}$ & & & $1(6.2 \%)$ \\
\hline \multicolumn{4}{|l|}{ Coexisting lung disease } \\
\hline Emphysema & & $1(4.2 \%)$ & $4(25.0 \%)$ \\
\hline $\begin{array}{l}\text { Usual interstitial } \\
\text { pneumonia }\end{array}$ & $1(2.3 \%)$ & $1(4.2 \%)$ & $4(25.0 \%)$ \\
\hline Pneumoconiosis & 0 & $2(8.3 \%)$ & \\
\hline Bronchiectasis & $3(6.8 \%)$ & $2(8.3 \%)$ & \\
\hline \multicolumn{4}{|l|}{ Underlying diseases } \\
\hline Diabetes & $1(2.3 \%)$ & $2(8.3 \%)$ & $1(6.2 \%)$ \\
\hline Malignancy ${ }^{\mathrm{b}}$ & $6(13.6 \%)$ & $3(12.5 \%)$ & \\
\hline Prior use of steroids $\mathrm{c}^{\mathrm{c}}$ & $1(2.3 \%)$ & $1(4.2 \%)$ & $2(12.5 \%)$ \\
\hline
\end{tabular}

CT: computed tomography

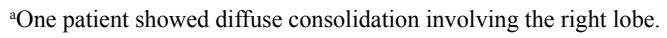

${ }^{b}$ Malignancy includes cancers of the stomach, colon, liver, breast, and lung, multiple myeloma, and leukaemia.

'Prior use of steroids means the use of steroids within 1 year prior to CT examination

The second most common finding was a mass greater than 3 $\mathrm{cm}$ in diameter in 24 cases (28.6\%), of which nine cases had daughter nodules (37.5\%). Mass lesions were spiculated in 14 cases $(58.3 \%)$, lobulated in nine cases (37.5\%), and smooth in one case (4.2\%). Masses ranged in diameter from 3.3 to 5.7 $\mathrm{cm}$. Further, sixteen cases showed nonsegmental consolidation (19.0\%), with daughter nodules appearing in four of those cases. These sixteen patients, with consolidation mimicking a pneumonia-like infiltrate that did not clear on antibiotic therapy and a negative sputum result for M. tuberculosis, were diagnosed based on the PTNB findings.

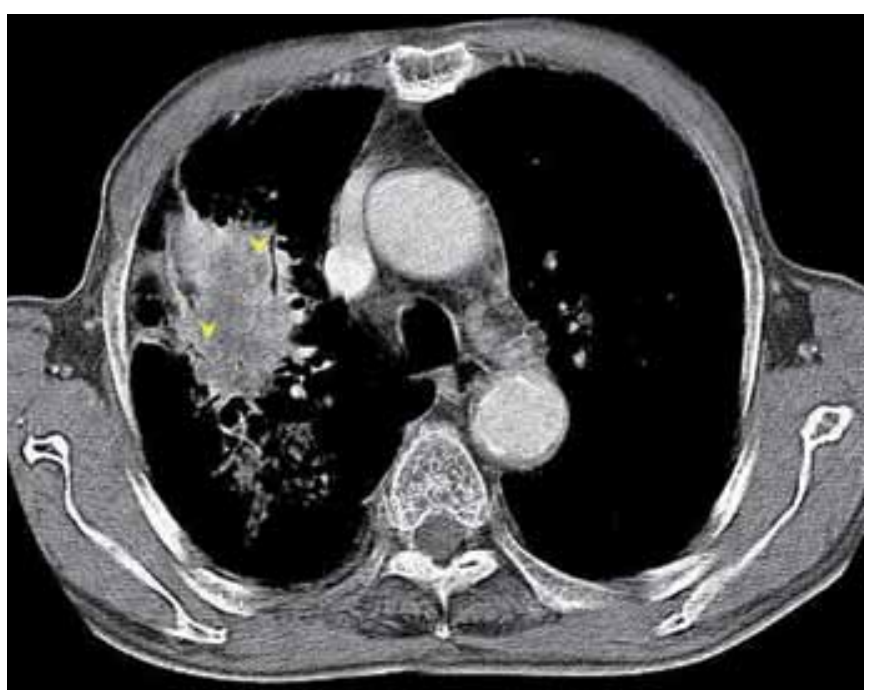

FIG. 1. TB appearing as consolidation in a 74-year-old man with emphysema. In the mediastinum window of the CT scan, there are some low-density areas (arrowheads) within the consolidation in the right upper lobe that did not improve despite antibiotic therapy. Fluoroscopy-guided PTNB revealed tuberculosis

Abnormal $\mathrm{CT}$ findings and coexisting pulmonary diseases in the 84 patients are summarized in Table 1. Cavitation was present in three nodules, eight masses, and two consolidative lesions. Mediastinal lymph node enlargement was present in two cases, one case with a mass and the other with a nodule, and pleural effusion was present in one nodule case (not shown). One patient had eccentric calcification within both a nodule and mass.

Tuberculosis lesions in 52 patients $(61.9 \%)$ were located in the upper lobes, while 24 patients had lesions in the lower lobes $(28.6 \%)$, seven patients $(8.3 \%)$ in the right middle lobe, and one patient in the right lung (1.2\%), which manifested as consolidation.

With regard to coexisting pulmonary diseases, six patients had coexisting emphysema. This included one patient with a nodule and one patient with a mass, while the remaining four cases appeared in patients with CT features of nonsegmental consolidation (Figure 1). Usual interstitial pneumonia (UIP) was present in one case with a mass and in four cases with consolidative TB lesions (Figure 2). Pneumoconiosis was present in two cases with mass lesions (Figure 3). There were no significant coexisting lung diseases in the other patients.

Seventeen patients $(20.2 \%)$ had an underlying disease associated with an immunocompromised state. These conditions included: diabetes mellitus, lung cancer, stomach cancer, colon cancer, breast cancer, hepatocellular carcinoma, acute myelocytic leukaemia, multiple myeloma, or steroid therapy within 1 year from the $\mathrm{CT}$ examination.

Complications after PTNB included seven patients who required thoracic drainage for pneumothorax and 16 patients 
who had haemoptysis immediately after the procedure that subsequently resolved after conservative treatment.

\section{DISCUSSION}

Hadlock et al. (6) indicated that atypical manifestations of TB (excluding the primary TB pattern in adults) are prevalent in nearly $8 \%$ of cases. However, recent studies have suggested that unusual radiographic manifestations of TB may be encountered in as many as one third of adult-onset TB cases, which may seriously delay treatment. Post-primary TB can be considered unusual for two reasons. First, it is confined to

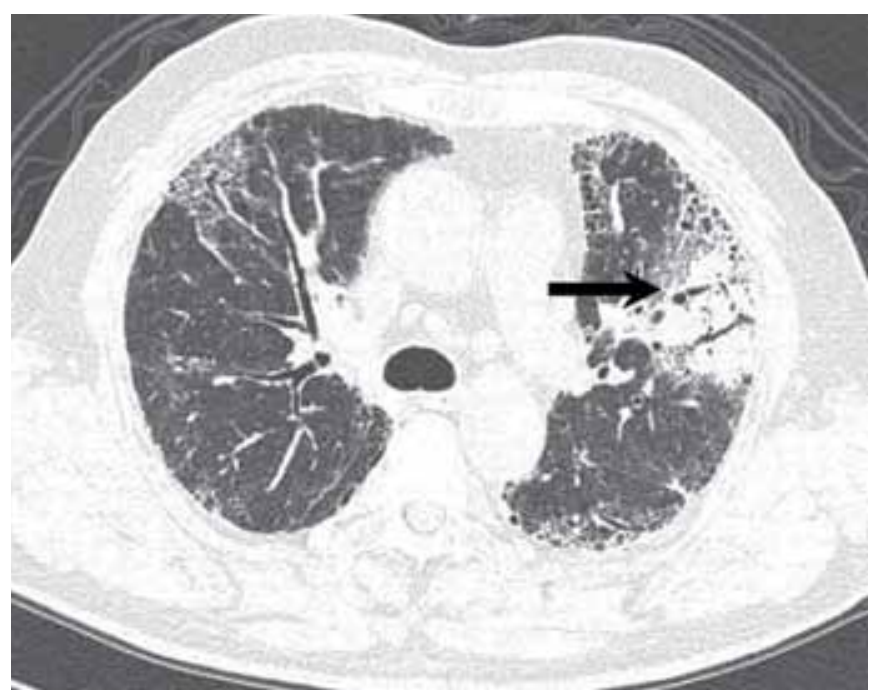

FIG. 2. TB appearing as nonsegmental consolidation in a 73-yearold man with UIP (the patient was diagnosed with UIP 5 years prior). A high-resolution CT scan obtained at the subcarinar level shows nonsegmental consolidation in the left upper lobe (arrow). The consolidation in the left upper lobe had not improved despite antibiotic therapy. Therefore, PTNB was conducted and it was diagnosed as TB the basal segments of lower lobes, the right middle lobe or lingular, or the anterior segment of an upper lobe and second, it can manifest as a mass-like density that mimics carcinoma, tuberculoma, or reversible multiple cysts (7-9). Atypical manifestations of pulmonary TB are common in patients with impaired host immunity (10-12) and are now considered to be manifestations of primary TB $(13,14)$.

Pulmonary TB can be a cause of nodular, or mass-like, lung opacities in asymptomatic patients whose sputum smears or cultures may be negative for mycobacteria (15). Pulmonary $\mathrm{TB}$ is one of the most common benign lesions that requires differentiation from lung cancer $(16,17)$. Even though our series was not specific, 26 of 44 punctured nodules and 14 of 24 masses had an irregular or spiculate contour. These were initially considered malignant. Due to the overlapping features of tuberculomas and peripheral lung cancer, PTNB should be performed to confirm the diagnosis (18). 18F-FDG PET can improve diagnostic accuracy by allowing differentiation of solitary pulmonary nodules (19); however, ${ }^{18} \mathrm{~F}-\mathrm{FDG}$ is a nontumour-specific tracer, and all tissues with high-glucose metabolism can show high uptake $(20,21)$.

When post-primary TB involves the anterior segment of an upper lobe or basilar segment of a lower lobe, radiography may sometimes misdiagnose it as another bacterial infection (22). These lesions are initially refractory to antibiotic therapy, thereby making the correct diagnosis challenging. In the present study, 16 cases of pulmonary TB resembled pneumonia on chest radiographs or CT scans. We suggest that if nonsegmental consolidations are refractory to initial antibiotic treatment, the next step should be biopsy.

Silicosis and TB are known to be associated. TB rates in subjects with advanced simple silicosis, in an elevated background TB setting, can be very high, up to three-fold higher than those in the same workforce without silicosis (23); further, risk increases with the severity of silicosis. In the present study, percutaneous biopsy was very useful for diagnosing
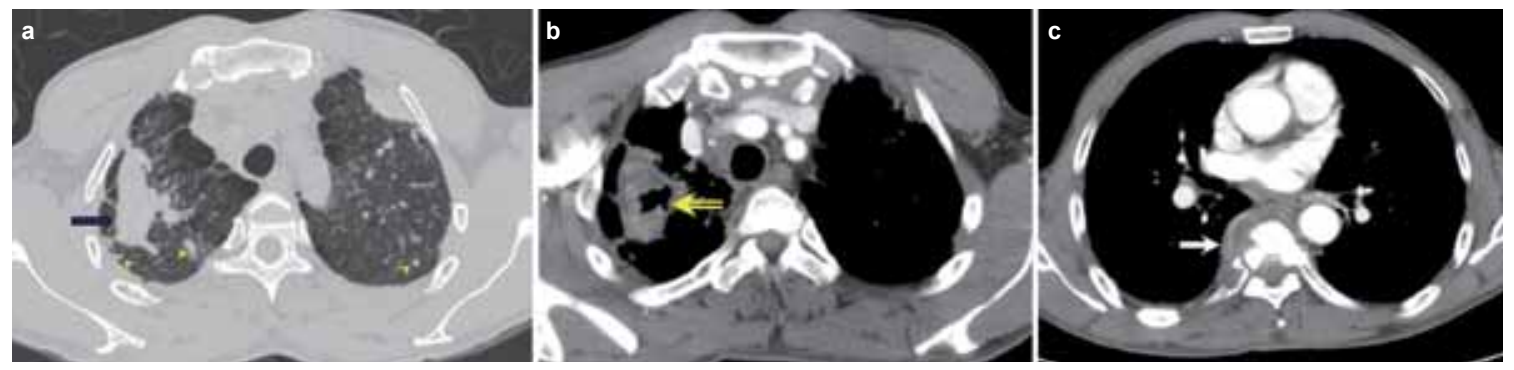

FIG. 3. a-c. TB appearing as a cavitating mass in a 57-year-old man with pneumoconiosis. A 5-cm conglomerated mass (arrow) in the right upper lobe and multiple small nodules (arrowheads) bilaterally in the high-resolution CT scan (a) taken 10 months before admission. This patient had been given a diagnosis of coal worker's pneumoconiosis 5 years prior. On admission, the mediastinum window setting (b) showed cavitation (open arrow) of the mass in the right upper lobe with growth of the mass $(6.5 \mathrm{~cm}$ in diameter) and the osteolytic T7 (c, arrow) with paravertebral soft tissue formation on admission. CT-guided biopsy was performed to differentiate between lung cancer with bone metastasis versus pulmonary TB with spine involvement. The final diagnosis was tuberculosis. This case illustrates cavitation and growth of progressive massive fibrosis after infection with $M$. tuberculosis 
silicotuberculosis because these cases had negative results in the microbiologic analysis.

Tuberculosis and emphysema, or UIP, may co-exist in the same patient (24-26) and can be a significant burden in terms of morbidity and mortality. In our study, four cases showed nonsegmental consolidation and one case showed a spiculate $3.2 \mathrm{~cm}$ mass, which was consistent with those in a previous study (25). CT features of TB in UIP patients showed that TB in patients with severe emphysema manifested as patchy consolidation. The cause of these findings in UIP and emphysema patients does not necessarily mean that the TB involved the normal lung parenchyma. The tuberculous infiltration may have affected already damaged and destructed lung parenchyma, such as the honeycomb-like parenchyma caused by emphysema. This may result in pneumonia-like consolidation and lack of bronchogenic evidence of TB-like centrilobular nodules or the tree-in-bud pattern. Another reason for the atypical radiologic finding of TB in association with UIP is that UIP patients are often prescribed an immunosuppressant such as steroids, which increases susceptibility to mycobacterial infection (11).

Failure to demonstrate tubercle bacilli in sputum, or other secretions, cannot rule out the diagnosis of TB. This is because the lesions are occasionally not in contact with the bronchial tree, or the number of AFBs in sputum smears is low. Therefore, in areas where TB is endemic, mycobacterial infection should be considered when processing aspirates obtained after PTNB of peripheral lung opacities, even if the results of sputum cultures or bronchoscopic stains and cultures are negative for mycobacteria $(27,28)$.

A limitation of our study is its retrospective design. Subjects were patients who had been diagnosed with TB based on histopathological findings of biopsy specimens and PCR results for M. tuberculosis. This bias could have affected the results of the study. Another limitation is the absence of DNA fingerprinting to demonstrate recent or remote infection, or to differentiate between primary or post-primary TB. Finally, we did not evaluate for interobserver variations, but analysed the CT findings by consensus. Further studies with a prospective design and a large patient population, comprising UIP or emphysema patients, are required to confirm our findings.

In conclusion, the most common finding on CT scans for patients with pulmonary TB confirmed via PTNB was a nodule or a mass. It is strongly helpful in the diagnosis of pulmonary TB to take a PTNB in patients showing airspace consolidation with underlying UIP or emphysema mimicking bacterial pneumonia, as well as a mass with pneumoconiosis mimicking lung malignancy.

Ethics Committee Approval: Ethics committee approval was received for this study (IRB File No is AS12148-002.)

Informed Consent: Written informed consent was obtained from patients who participated in this study.
Peer-review: Externally peer-reviewed.

Author contributions: Concept - J.Y.C., K.Y.L.; Design - K.Y.L., M.K., E.K., Y.W.O.; Supervision - K.Y.L., S.H.L., B.K.S.; Resource - S.H.L., B.K.J.; Materials - J.Y.C., Y.H.O., M.Y.K., K.Y.L.; Data Collection\&/or Processing K.Y.L., J.Y.C., M.K.; Analysis\&/or Interpretation - K.Y.L., J.Y.C.; Literature Search - K.Y.L., J.Y.C., M.K.; Writing - J.Y.C.; Critical Reviews - K.Y.L., B.K.J.

Conflict of Interest: No conflict of interest was declared by the authors.

Financial Disclosure: This study was supported by Research Grant of Korea University (K0932161), and by Dong-Kook pharmaceutical.

\section{REFERENCES}

1. Andreu J, Cáceres J, Pallisa E, Martinez-Rodriguez M. Radiological manifestations of pulmonary tuberculosis. Eur J Radiol 2004;51:13949. [CrossRef]

2. Diagnostic Standards and Classification of Tuberculosis in Adults and Children. This official statement of the American Thoracic Society and the Centers for Disease Control and Prevention was adopted by the ATS Board of Directors, July 1999. This statement was endorsed by the Council of the Infectious Disease Society of America, September 1999. Am J Respir Crit Care Med 2000;161:1376-95. [CrossRef]

3. Jeong YJ, Lee KS. Pulmonary tuberculosis: up-to-date imaging and management. AJR Am J Roentgenol 2008;191:834-44. [CrossRef]

4. Narayana Reddy RA, Narayana SM, Shariff S. Role of fine-needle aspiration cytology and fluid cytology in extra-pulmonary tuberculosis. Diagn Cytopathol 2013;41:392-8. [CrossRef]

5. Shachor Y, Schindler D, Siegal A, Lieberman D, Mikulski Y, Bruderman I. Increased incidence of pulmonary tuberculosis in chronic interstitial lung disease. Thorax 1989;44:151-3. [CrossRef]

6. Hadlock FP, Park SK, Awe RJ, Rivera M. Unusual radiographic findings in adult pulmonary tuberculosis. AJR Am J Roentgenol 1980;134:1015-8. [CrossRef]

7. Miller WT, MacGregor RR. Tuberculosis: Frequency of unusual radiographic findings. AJR Am J Roentgenol 1978;130:867-75. [CrossRef]

8. Lee KS, Song KS, Lim TH, Kim PN, Kim IY, Lee BH. Adult-onset pulmonary tuberculosis: findings on chest radiographs and CT scans. AJR Am J Roentgenol 1993;160:753-8. [CrossRef]

9. Palmer PE. Pulmonary tuberculosis-usual and unusual radiographic presentations. Semin Roentgenol 1979;14:204-43. [CrossRef]

10. Van Dyck P, Vanhoenacker FM, Van den Brande P, De Schepper AM. Imaging of pulmonary tuberculosis. Eur Radiol 2003;13:1771-85. [CrossRef]

11. Ikezoe J, Takeuchi N, Johkoh T, Kohno N, Tomiyama N, Kozuka T, et al. CT appearance of pulmonary tuberculosis in diabetic and immunocompromised patients: comparison with patients who had no underlying disease. AJR Am J Roentgenol 1992;159:1175-9. [CrossRef]

12. Morris JT, Seaworth BJ, McAllister CK. Pulmonary tuberculosis in diabetics. Chest 1992;102:539-41. [CrossRef]

13. Jones BE, Ryu R, Yang Z, Cave MD, Pogoda JM, Otaya M, et al. Chest radiographic findings in patients with tuberculosis with recent or remote infection. Am J Respir Crit Care Med 1997;156:1270-3. [CrossRef]

14. Geng E, Kreiswirth B, Burzynski J, Schluger NW. Clinical and radiographic correlates of primary and reactivation tuberculosis: a molecular epidemiology study. JAMA 2005;293:2740-5. [CrossRef]

15. Ferreirós J, Bustos A, Merino S, Castro E, Dorao M, Crespo C. Transthoracic needle aspiration biopsy: value in the diagnosis of mycobacterial lung opacities. J Thorac Imaging 1999;14:194-200. [CrossRef] 
16. Zwirewich CV, Vedal S, Miller RR, Müller NL. Solitary pulmonary nodule: high-resolution CT and radiologic-pathologic correlation. Radiology 1991;179:469-76. [CrossRef]

17. Murayama S, Murakami J, Hashimoto S, Torii Y, Masuda K. Noncalcified pulmonary tuberculomas: CT enhancement patterns with histological correlation. J Thorac Imaging 1995;10:91-5. [CrossRef]

18. Thiessen R, Seely JM, Matzinger FR, Agarwal P, Burns KL, Dennie $\mathrm{CJ}$, et al. Necrotizing granuloma of the lung: imaging characteristics and imaging-guided diagnosis. AJR Am J Roentgenol 2007;189:1397401. [CrossRef]

19. Li Y, Su M, Li F, Kuang A, Tian R. The value of (1)(8)F-FDG-PET/CT in the differential diagnosis of solitary pulmonary nodules in areas with a high incidence of tuberculosis. Ann Nucl Med 2011;25:804-11. [CrossRef]

20. Zhuang H, Alavi A. 18-fluorodeoxyglucose positron emission tomographic imaging in the detection and monitoring of infection and inflammation. Semin Nucl Med 2002;32:47-59. [CrossRef]

21. Rosenbaum SJ, Lind T, Antoch G, Bockisch A. False-positive FDG PET uptake-the role of PET/CT. Eur Radiol 2006;16:1054-65. [CrossRef]
22. Yoon JY, Lee IJ, Im HJ, Lee K, Lee Y, Bae SH. CT findings in apical versus basal involvement of pulmonary tuberculosis. Diagn Interv Radiol 2013;19:85-90.

23. Cowie RL. The epidemiology of tuberculosis in gold miners with silicosis. Am J Respir Crit Care Med 1994;150:1460-2. [CrossRef]

24. Chakrabarti B, Calverley PM, Davies PD. Tuberculosis and its incidence, special nature, and relationship with chronic obstructive pulmonary disease. Int J Chron Obstruct Pulmon Dis 2007;2:263-72.

25. Chung MJ, Goo JM, Im JG. Pulmonary tuberculosis in patients with idiopathic pulmonary fibrosis. Eur J Radiol 2004;52:175-9. [CrossRef]

26. Park SW, Song JW, Shim TS, Park MS, Lee HL, Uh ST, et al. Mycobacterial pulmonary infections in patients with idiopathic pulmonary fibrosis. J Korean Med Sci 2012;27:896-900. [CrossRef]

27. Silverman JF, Marrow HG. Fine needle aspiration cytology of granulomatous diseases of the lung, including nontuberculous Mycobacterium infection. Acta Cytol 1985;29:535-41.

28. Yuan A, Yang PC, Chang DB, Yu CJ, Lee LN, Wu HD, et al. Ultrasound guided aspiration biopsy for pulmonary tuberculosis with unusual radiographic appearances. Thorax 1993;48:167-70. [CrossRef] 